University of Wollongong

Research Online

SMART Infrastructure Facility - Papers

Faculty of Engineering and Information

Sciences

$1-1-2016$

A fuzzy rough sets-based multi-agent analytics framework for dynamic supply chain configuration

Nagesh Shukla

University of Wollongong, nshukla@uow.edu.au

Senevi Kiridena

University of Wollongong, skiriden@uow.edu.au

Follow this and additional works at: https://ro.uow.edu.au/smartpapers

Part of the Engineering Commons, and the Physical Sciences and Mathematics Commons

Research Online is the open access institutional repository for the University of Wollongong. For further information contact the UOW Library: research-pubs@uow.edu.au 


\title{
A fuzzy rough sets-based multi-agent analytics framework for dynamic supply chain configuration
}

\begin{abstract}
Considering the need for more effective decision support in the context of distributed manufacturing, this paper develops an advanced analytics framework for configuring supply chain networks. The proposed framework utilizes a distributed multi-agent system architecture to deploy fuzzy rough sets-based algorithms for knowledge elicitation and representation. A set of historical sales data, including network node-related information, is used together with the relevant details of product families to predict supply chain configurations capable of fulfilling desired customer orders. Multiple agents such as data retrieval agent, knowledge acquisition agent, knowledge representation agent, configuration predictor agent, evaluator agent and dispatching agent are used to help execute a broad spectrum of supply chain configuration decisions. The proposed framework considers multiple product variants and sourcing options at each network node, as well as multiple performance objectives. It also captures decisions that span the entire supply chain simultaneously and, by implication, represents multiple network links. Using an industry test case, the paper demonstrates the effectiveness of the proposed framework in terms of fulfilling customer orders with lower production and emissions costs, compared to the results generated using existing tools.
\end{abstract}

\section{Keywords}

multi, configuration, sets, fuzzy, rough, chain, supply, dynamic, framework, analytics, agent

\section{Disciplines}

Engineering | Physical Sciences and Mathematics

\section{Publication Details}

Shukla, N. \& Kiridena, S. (2016). A fuzzy rough sets-based multi-agent analytics framework for dynamic supply chain configuration. International Journal of Production Research, 54 (23), 6984-6996. 


\title{
A Fuzzy Rough Sets-based Multi-agent Analytics Framework for Dynamic Supply Chain Configuration
}

\author{
Nagesh Shukla $^{1^{*}}$, Senevi Kiridena ${ }^{2}$ \\ ${ }^{1}$ SMART Infrastructure Facility, Faculty of Engineering and Information Sciences, \\ University of Wollongong, Wollongong, NSW 2522, Australia \\ ${ }^{2}$ School of Mechanical, Materials and Mechatronics Engineering, Faculty of Engineering and \\ Information Sciences, University of Wollongong, Wollongong, NSW 2522, Australia \\ ${ }^{*}$ Corresponding Author
}

\begin{abstract}
Considering the need for more effective decision support in the context of distributed manufacturing, this paper develops an advanced analytics framework for configuring supply chain networks. The proposed framework utilizes a distributed multi-agent system architecture to deploy fuzzy rough sets-based algorithms for knowledge elicitation and representation. A set of historical sales data, including network node-related information, is used together with the relevant details of product families to predict supply chain configurations capable of fulfilling desired customer orders. Multiple agents such as data retrieval agent, knowledge acquisition agent, knowledge representation agent, configuration predictor agent, evaluator agent and dispatching agent are used to help execute a broad spectrum of supply chain configuration decisions. The proposed framework considers multiple product variants and sourcing options at each network node, as well as multiple performance objectives. It also captures decisions that span the entire supply chain simultaneously and, by implication, represents multiple network links. Using an industry test case, the paper demonstrates the effectiveness of the proposed framework in terms of fulfilling customer orders with lower production and emissions costs, compared to the results generated using existing tools.
\end{abstract}

Keyword: manufacturing processes, multi-agent systems, supply chain

\section{Introduction}

With the emerging technological advancements that enable distributed manufacturing come opportunities for businesses to explore new sources of competitive advantage. To successfully explore such opportunities organisations must possess the capacity to make robust decisions. In an environment where distributed manufacturing networks operate at a global scale, due to the diverse range of factors that need to be considered and the pace at which they change, the need for effective decision-making becomes even greater (Olhager et al., 2015; Wang and Chan, 2010). Supply chain (SC) management research can make a significant contribution to advance this cause by way of developing suitable decision support systems (DSS).

The call for more effective decision support in the context of distributed manufacturing is justified on a number of accounts. First, shifting competitive dynamics and ongoing industry consolidations exert increasing pressure on manufacturing enterprises to integrate into global SC networks (Wang and Chan, 2010; Shen et al., 2006). Second, addressing the trade-offs 
concerning the need for upholding the commercial interests (opportunism) of partner entities while being able to optimise the performance of the entire SC network demands innovative approaches to SC management (Mustafee et al., 2012; Qu et al., 2010). Third, current developments on the social, economic and technological fronts that enhance the accessibility to numerous forms of data (from multiple sources) create new opportunities for such data to be productively used for decision support (Mortenson et al., 2015; Bose, 2009). The work pursued through this paper is motivated by the need for responding to the challenges and opportunities presented by these developments (Fawcett and Waller, 2014).

To this end, this paper develops an advanced analytics framework that can be used to support SC configuration decisions. The paper first presents a summary account of the state-of-the-art in SC configuration decision support, including major limitations of the existing approaches. It then introduces the proposed agent-based architecture, accompanied by a detailed description of its constituent elements. Application of this framework is then illustrated using a test case and sample data drawn from the literature. The paper concludes with an assessment of the efficacy of the proposed framework in light of the results generated and further opportunities for improving and extending the framework.

\section{Literature Review}

There is a substantive body of knowledge to inform SC network design decisions such as facilities location, capacity planning and supplier selection (Olhager et al., 2015; Meixell and Gargeya, 2005). This body of knowledge is complemented by the extensive work undertaken in the areas of mathematical optimisation and simulation modelling (Acar et al., 2010; Iannone et al., 2007; Shen et al., 2006; Terzi and Cavalieri, 2004). By comparison, much less attention has been paid to research concerning SC network configuration decisions. The interest in configuration decisions arises out of the situation where, in response to the changes in the internal and external business environments, organisations need to assess the alignment between their competitive priorities and SC structure on an ongoing basis, to remain competitive. Inevitably, any consequent adjustments of network nodes or links do affect the performance of the whole SC. Most of the available analytical models and mathematical optimisation techniques do not have the capacity to account for such adaptive and dynamic dimensions of SC network configuration (Zhang et al., 2009; Akanle and Zhang, 2008; Piramuthu, 2005).

Supply chain configuration decisions involve the determination of network nodes and links to meet a set of product-related functional, as well as organisational, objectives while recognising the adaptive nature of SC networks and being able to respond to the dynamics of the business environment. Therefore, configuration decisions are still considered as those concerning the SC network structure; however, compared to design decisions, they transpire more frequently. Thus, the aim of optimisation is to generate a number of alternative (feasible) configurations decision-makers can choose from to suit a given set of circumstances, instead of a single globally-optimised configuration which is often the case in SC design.

Most of the DSS developed within the context of SC design have been based on the assumption that once a network is configured to suit a given product architecture it would remain the same for the foreseeable future (Blackhurst et al., 2005; Huang et al., 2005). Given that configuration, subsequent research efforts generally aim at optimising operations performance. Research focusing on static network designs has also employed analytical methods (both deterministic and stochastic) such as mixed-integer programming and multi- 
objective optimisation algorithms, as well as systems modelling and simulation to derive the best globally-optimised network configuration (Olhager et al., 2015; Mustafee et al., 2012; Beamon, 1998). In most cases, these optimisations have been achieved against a single performance objective such as cost (Meixell and Gargeya, 2005; Beamon, 1998).

However, a number of authors have pointed out that such an approach is insufficient to provide effective support required for robust decision-making in a dynamic global environment. For instance, Akanle and Zhang (2008) emphasized the need for recognising the evolving nature of SCs when developing methodologies to address the configuration problem. Olhager et al. (2015), as well as Meixell and Gargeya (2005), noted the need for extending current decision support models to capture multiple decisions and multiple objectives across the SC in their entirety, while accounting for contingency factors. Similarly, Piramuttu (2005) called for studies that deal with the dynamic configuration of SCs and "extending the research focus to handle more stages, several nodes in each stage and variability [in order size or type]” (p. 229). More broadly, the literature has also highlighted the limitations of existing analytical methods used for dealing with the above challenges $(\mathrm{Qu}$ et al., 2015; Long and Zhang, 2014; Mustafee et al., 2012).

A few authors have attempted to comprehensively tackle the above challenges using novel methodological approaches. For example, Akanle and Zhang (2008) developed a multi-agent system to optimise configuration decisions considering anticipated changes in customer demand, as well as ongoing adjustments in the resource profiles of SC entities and their operational environment. Troung and Azadivar (2005) proposed a SC model builder combining genetic algorithms, mixed integer programing and discrete-event simulation to address multiple configuration decisions (of both qualitative and quantitative nature) simultaneously. Piramuttu (2005) applied machine learning algorithms to identify the nodes of a SC network that best aligns with a given combination of order attributes, which was shown to deliver better SC-wide performance. The current status of SC design/configuration research, as revealed through the literature review, is summarised in Figure 1 below with the research gaps this paper aims to address largely falling in quadrant 4 (Q4).

\begin{tabular}{|c|c|c|c|}
\hline & \multicolumn{2}{|c|}{ Spatial dimension } \\
\hline & & Narrow scope (reductionist) & Broad scope (holistic) \\
\hline \multirow{2}{*}{ 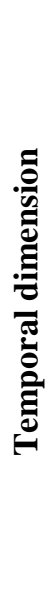 } & 荀 & $\begin{array}{l}\text { - } \text { assume stable entities and relationships } \\
\text { - driven by product architectures and } \\
\text { performance improvement goals } \\
\text { - } \text { focus on single-objective optimisation } \\
\text { - well-researched; mature knowledge base } \\
\text { - a wide range of tools and techniques are } \\
\text { available to choose from }\end{array}$ & $\begin{array}{l}\text { - } \text { assume stable entities and networks } \\
\text { - driven by product architectures and } \\
\text { competitive priorities } \\
\text { - } \text { focus on global optimisation, few objectives } \\
\text { - } \text { and centralised external control } \\
\text { - } \text { limited-research; emerging knowledge base } \\
\text { some tools and techniques }\end{array}$ \\
\hline & 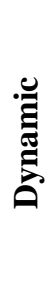 & $\begin{array}{l}\text { - } \text { assume evolving entities and relationships Q3 } \\
\text { - driven by product architectures and } \\
\text { - } \text { technology platforms } \\
\text { - } \text { and distributed control } \\
\text { - limited-research; emerging knowledge base } \\
\text { - } \text { some tools and techniques }\end{array}$ & $\begin{array}{l}\text { - } \text { assume evolving entities and networks } \\
\text { driven by competitive priorities, } \\
\text { organisational capabilities, product } \\
\text { architectures and technology platforms } \\
\text { - focus on scenario-based optimisation, } \\
\text { multiple objectives and self-control } \\
\text { - little research; no tools and techniques }\end{array}$ \\
\hline
\end{tabular}

Figure 1: State-of-the-art in SC configuration research 
The work reported in this paper aims to contribute to this stream of literature by addressing the problem of generating a suit of network configurations capable of delivering a given mix of customer orders that could be evaluated against multiple performance goals. In doing so, we extend the work of Akanle and Zhang (2008), Troung and Azadivar (2005) and Piramuttu (2005) by way of enhancing their scope and methodology. Previous studies have employed individual methods such as machine learning and genetic algorithms, as well as agent-based approaches, to account for the dynamic and adaptive dimensions of the network configuration problem. Agent-based approaches in particular have been found to be useful in representing the distinctive behaviour of individual entities within the SC network (Dai et al., 2014; Long and Zhang, 2014; Shen et al., 2006). Following our evaluation of the available tools to ascertain their capacity to meet the multiple requirements discussed above, we selected a fuzzy rough sets-based multi-agent approach. In the following section, we illustrate how this approach is used to solve the SC configuration problem.

\section{Multi-agent architecture for SC configuration}

In general, agents are defined as independent problem-solvers with capabilities for sensing and acting within their environment, which include communicating with other agents, to decide their course of action in an autonomous way (Monostori et al., 2006). The multi-agent system (MAS) architecture proposed in this paper is based on: (i) knowledge handling by agents; (ii) a data-based intuitive analytics approach; and (iii) a number of similarly coordinating/communicating agents. As such, it comprises a group of agents namely: data retrieval agent, knowledge acquisition agent, knowledge representation agent, configuration predictor agent, SC configuration evaluator agent and dispatcher agent. Each of these agents is assigned to perform specific tasks that facilitate SC configuration decisions. Collectively these agents provide SC configuration solutions to satisfy a mix of customer orders, which are driven by chosen competitive priorities, while meeting multiple performance objectives (e.g. total cost, $\mathrm{CO}_{2}$ emissions and total delivery time, among others).

Due to the advancement of data acquisition and storage technologies, SC organisations are typically capturing and storing various datasets related to product sourcing, design, manufacturing, logistics and field performances. These datasets can potentially be used to inform SCM decisions. In this paper, we demonstrate the use of some historical product data sets for generating SC configurations that are aligned with an organisation's competitive priorities. We employ an innovative set of techniques from fuzzy rough sets theory (FRST) (Cornelis et al., 2010; 2008) to mine rules and associations from such historical datasets. Rough set theory (RST) was first introduced by Pawlak (1982) for mining data based on the information theory-based approximations. It allows an effective mechanism for distinguishing objects based on their attribute values. These are then used to form simple intuitive rules (IF-THEN) for classifying objects. FRST is a generalised form of RST where imprecision or vagueness is represented by membership degrees in fuzzy sets rather than discretisation for the real-valued attributes. More information about FRST is presented later in this section.

Figure 2 illustrates the MAS architecture proposed for supporting SC configuration decisions. 


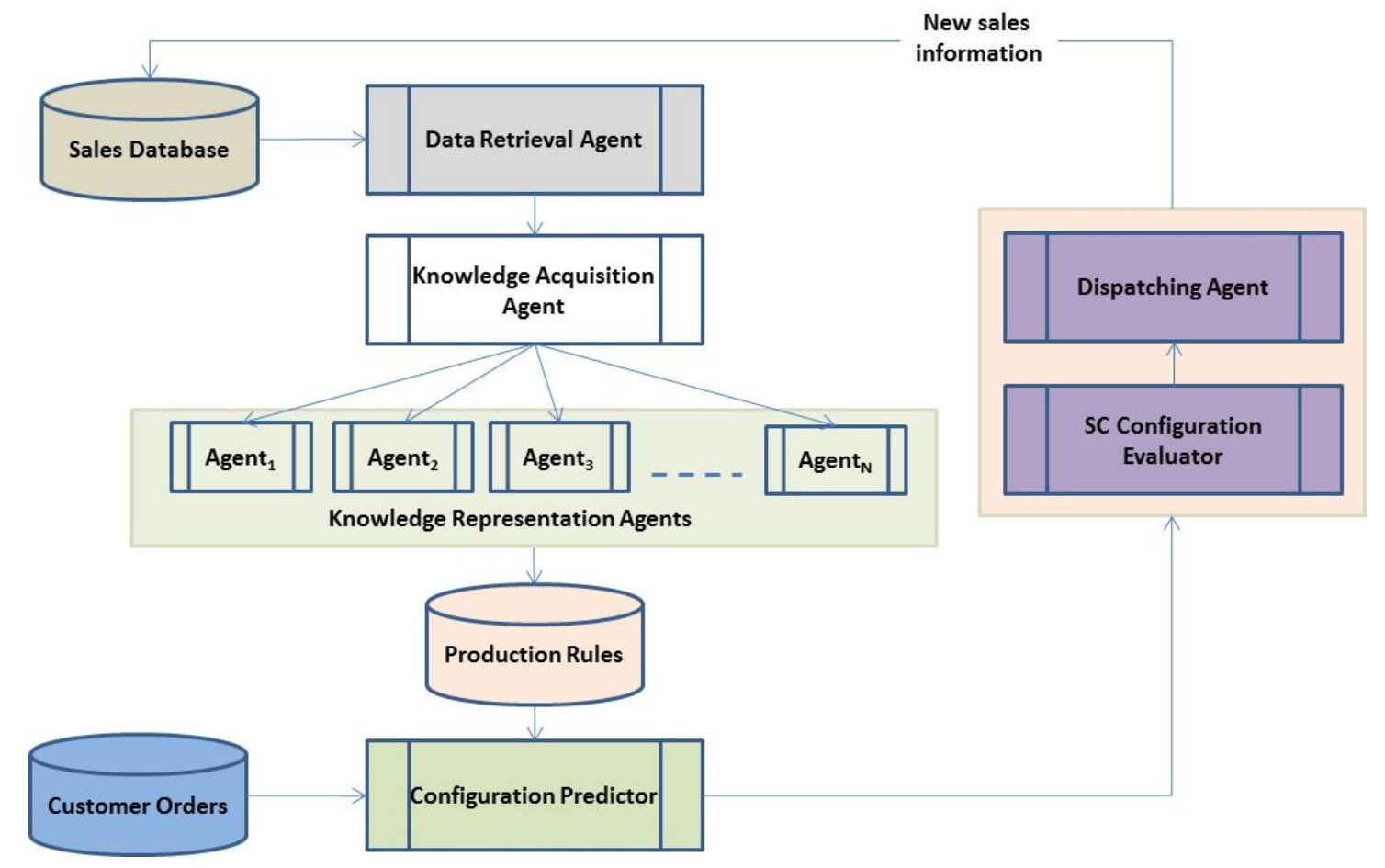

Figure 2: MAS Architecture for Dynamic SC Configuration

\subsection{Data retrieval agent}

The data retrieval agent retrieves historical product sales data and SC network node information. It pre-processes the raw records so that they can be used by the knowledge acquisition agent to prepare the dataset for analysis.

\subsection{Knowledge acquisition agent}

The knowledge acquisition agent performs some processing on the datasets retrieved by the data retrieval agent based on the FRST principles of discernibility, i.e., ability to distinguish objects based on measured attributes. The notations of FRST used throughout this paper are, therefore, introduced first. Let us assume that the dataset from the data retrieval agent is represented as $\mathcal{A}=(U, A \cup\{d\})$, where $U$ is a finite set of objects (data instances), $A$ is the finite non-empty set of attributes $(a)$ and $d$ is the decision attribute such that $d \notin A$. Mathematically, $a: U \rightarrow V_{a} \forall a \in A$ and $V_{a}$ is the set of values that an attribute $a$ can take. Decision variable $d$ can take only nominal/categorical values. The indiscernibility relations are established based on decision system $\mathcal{A}$. To model indiscernibility in decision classes (Bindiscernibility), an equivalence relation $R_{B}$ is defined for RST for set $B \subseteq A$ :

$$
R_{B}(x, y)=\left\{(x, y) \in U^{2} \mid a \in B, a(x)=a(y)\right\}
$$

According to this equivalence relation, if $(x, y) \in R_{B}(x, y)$, then $x$ and $y$ have exactly the same values for attributes in $B$. In the case of decision system $\mathcal{A}=(U, A \cup\{d\})$, equivalence classes are defined based on the designated attribute called the decision attribute. These equivalence classes are represented as $[x]_{R_{d}}$. 
However, in FRST, fuzzy indiscernibility relation is used to determine the degree to which two objects are indiscernible. The relation $R$ is assumed to be reflexive (Eqn. 2) and symmetric (Eqn. 3) with further tolerance conditions (Eqn. 4) imposed as needed.

That is,

$$
\begin{aligned}
& R(x, x)=1 \forall x \in U \\
& R(x, y)=R(y, x) \forall x, y \in U \\
& \mathcal{T}(R(x, y), R(y, z)) \leq R(x, z) \quad \forall x, y, z \in U
\end{aligned}
$$

where, the relation $R$ is called $\mathcal{T}$-equivalence relation and $\mathcal{T}($.$) is a given triangular norm$ operator.

In FRST, approximate equality relation $(R)$ for objects is measured instead of indiscernibility used in RST (Eqn. 1). To create fuzzy $B$-indiscernibility relation or approximate equality relation for $B \subseteq A$ with respect to quantitative attribute $a \in A$ is defined by $R_{a}$. According to Jensen and Shen (2009), this relation is typically represented as:

$$
R_{a}(x, y)=\max \left(\min \left(\frac{a(y)-a(x)+\sigma_{a}}{\sigma_{a}}, \frac{a(x)-a(y)+\sigma_{a}}{\sigma_{a}}\right), 0\right)
$$

where, $\sigma_{a}$ is the standard deviation of attribute $a$. In the case of nominal attributes, $R(x, y)=$ 1 if $a(x)=a(y)$ and $R(x, y)=0$ otherwise. Therefore, fuzzy $B$-indiscernibility relation is represented by:

$$
R_{B}(x, y)=\mathcal{T}(\underbrace{R_{a}(x, y)}_{a \in B})
$$

where, $\mathcal{T}($.$) is a t$-norm.

The upper and lower approximation sets for fuzzy rough set theory is generally given by an implicator function $\mathcal{J}$ and a t-norm function $\mathcal{T}$ (Radzikowska and Kerre, 2002). The fuzzy lower and upper approximations are given by:

$$
\begin{aligned}
& \left(R_{B} \downarrow X\right)(y)=i n f_{x \in U} \mathcal{J}\left(R_{B}(x, y), X(x)\right) \\
& \left(R_{B} \uparrow X\right)(y)=\sup _{x \in U} \mathcal{T}\left(R_{B}(x, y), X(x)\right)
\end{aligned}
$$

where, $X$ is a fuzzy set in $U$.

In FRST, fuzzy $B$-positive regions are defined as the fuzzy set of objects in $U$ that can be unequivocally classified using conditional attributes $B$. The fuzzy $B$-boundary region is defined to be the fuzzy set of objects in $U$ that can potentially, but not certainly, be classified using conditional attributes $B$. The fuzzy positive region (POS) can be defined based on the fuzzy B-indiscernibility relations for $y \in U$,

$$
\operatorname{POS}_{B}(y)=\left(\bigcup_{x \in U} R_{B} \downarrow[x]_{R_{d}}\right)(y) .
$$

The fuzzy $B$-boundary region is defined as: 


$$
B N D_{B}(y)=\left(\bigcup_{x \in U} R_{B} \uparrow[x]_{R_{d}} \backslash \bigcup_{x \in U} R_{B} \downarrow[x]_{R_{d}}\right)(y) .
$$

Once the fuzzy B-indiscernibility relationships are established, the decision-relative discernibility matrix is defined. Mathematically, discernibility matrix $(n \times n)$ is defined as

$$
c_{i j}=\left\{\begin{array}{cc}
\left\{a \in A: 1-R_{B}\left(x_{i}, x_{j}\right) \leq \lambda_{i}\right\}, & \text { if } d\left(x_{i}\right) \neq d\left(x_{j}\right) \\
\varnothing & \text { otherwise }
\end{array}\right.
$$

where, $\lambda_{i}=\left(R_{B} \downarrow\left[x_{i}\right]_{d}\right)\left(x_{i}\right)$.

The continuous attributes in the decision system $\mathcal{A}=(U, A \cup\{d\})$ is discretised based on the rough set approach. For instance, for $a \in A$ and the sequence of values it can take is defined by $\left\{v_{1}^{a}, v_{2}^{a}, \ldots, v_{n_{a}}^{a}\right\}$ and $v_{1}^{a}<v_{2}^{a}<\cdots<v_{n_{a}}^{a}$, the set of cuts on $a$ is denoted by:

$$
C_{a}=\left\{\left(a, \frac{v_{1}^{a}+v_{2}^{a}}{2}\right),\left(a, \frac{v_{2}^{a}+v_{3}^{a}}{2}\right), \ldots,\left(a, \frac{v_{n_{a}-1}^{a}+v_{n_{a}}^{a}}{2}\right)\right\} .
$$

Therefore, the set of all possible cuts $\forall a \in A$ is represented as

$$
C_{A}=\bigcup_{a \in A} C_{a}
$$

The discretised values in the decision system are then passed on to the knowledge representation agent for knowledge representation based on rule induction.

\subsection{Knowledge representation agents}

Knowledge representation agents use the information received from the acquisition agent (historical datasets) to create knowledge in the form of production rules. Each knowledge representation agent works with this information to represent knowledge for each node in the SC network. One of the popular knowledge representation techniques is the production rules commonly represented as IF < condition> THEN <action>. Advantages of rule-based representation include their simplicity and ease of use: e.g. interpretation and manipulation.

The algorithm used in this paper for generating decision rules is similar to the generalized fuzzy rough set rule induction (Zhao et al., 2010) and QuickRules algorithm (Jensen et al., 2009a,b). The method proposed by Zhao et al. (2010) can be briefly described as (see Fig. 3a):

Step 1: General lower approximation sets are developed to deal with misclassification and perturbation;

Step 2: Discernibility matrix is computed based on the consistency degree; and

Step 3: Rules based on consistency degrees of the associated objects are evaluated.

This algorithm is applied by agents to generate production rules for each node (supplier, manufacturers, retailer, others) in a distributed manner. The distributed nature of knowledge representation agents is achieved through adopting a bottom-up approach (i.e. from individual SC nodes to configuring the whole SC). The resulting knowledge is stored in the production rules database for its use in SC configuration predictions. 


\subsection{Configuration predictor agent}

Configuration predictor agent takes two types of input: (i) customer orders and (ii) production rules. The production rules are used together with the customer order attributes to predict appropriate SC configurations that are aligned with the SC performance objectives considered (see Fig. 3b). In this paper, we have used time, SC cost and carbon emissions as the three main indicators to assess SC-level performance. The customer orders are generally represented by the time window that customers are prepared to wait and the type of product that has been requested. More information about customer order attributes and production rules is presented in the results and discussion section.

\subsection{SC configuration evaluator agent}

Once the SC configurations for a given mix of customer orders are generated, these configurations are evaluated against the three performance metrics identified above (see Section 3.4). The total SC cost is used together with the selling price of the product to compute the total profit generated for the organisation by following the SC configurations concerned. At this stage, decision-makers can also consider other indicators such as carbon emissions and total production time before selecting an appropriate SC configuration to fulfil the desired mix of customer orders (see Fig. 3c).

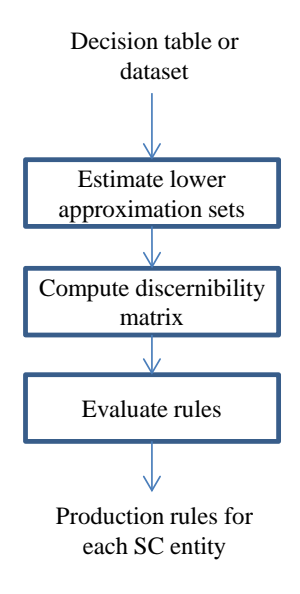

a

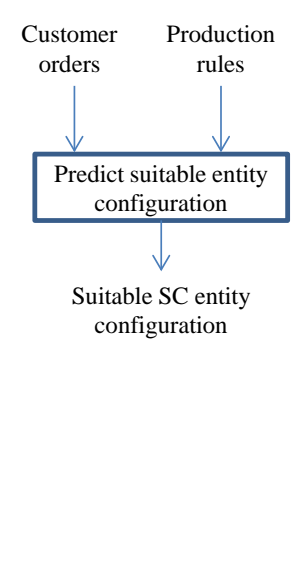

b

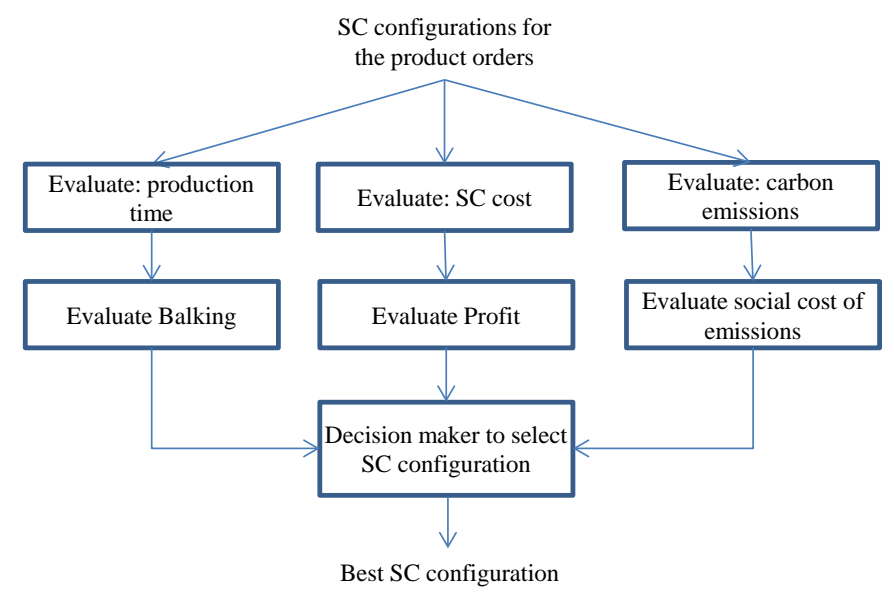

C

Figure 3: Agent actions for (a) knowledge representation agents, (b) configuration predictor, and (c) configuration evaluator agent

\subsection{Dispatching agent}

Based on the SC configuration decided by the configuration evaluator agent, subject to decision-makers preferences, the customer order is fulfilled. The dispatching agent sends information about the order and SC configuration to the relevant nodes in the SC network.

\section{Numerical Example}

In this section, we illustrate the implementation of the proposed framework using a popular example adapted from literature (Akanle and Zhang, 2008; Huang et al., 2005; Troung and 
Azadivar, 2005). In this example, the SC network considered involves the production of two product variants; Laptop-CD and Laptop-DVD. Both of these products require similar components and sub-assemblies until they reach the final stage of assembly where either CD or DVD subassembly is used. Figure 4 illustrates the structure of the SC network. We have adapted the example to suit the purpose of our paper with the changes listed below.

1. Both Laptop-CD and Laptop-DVD can be sold in all markets

2. There can be multiple suppliers with varying profiles (attributes) available to produce the required parts within the SC

3. Multiple assembly plants are available to produce subassemblies within the SC

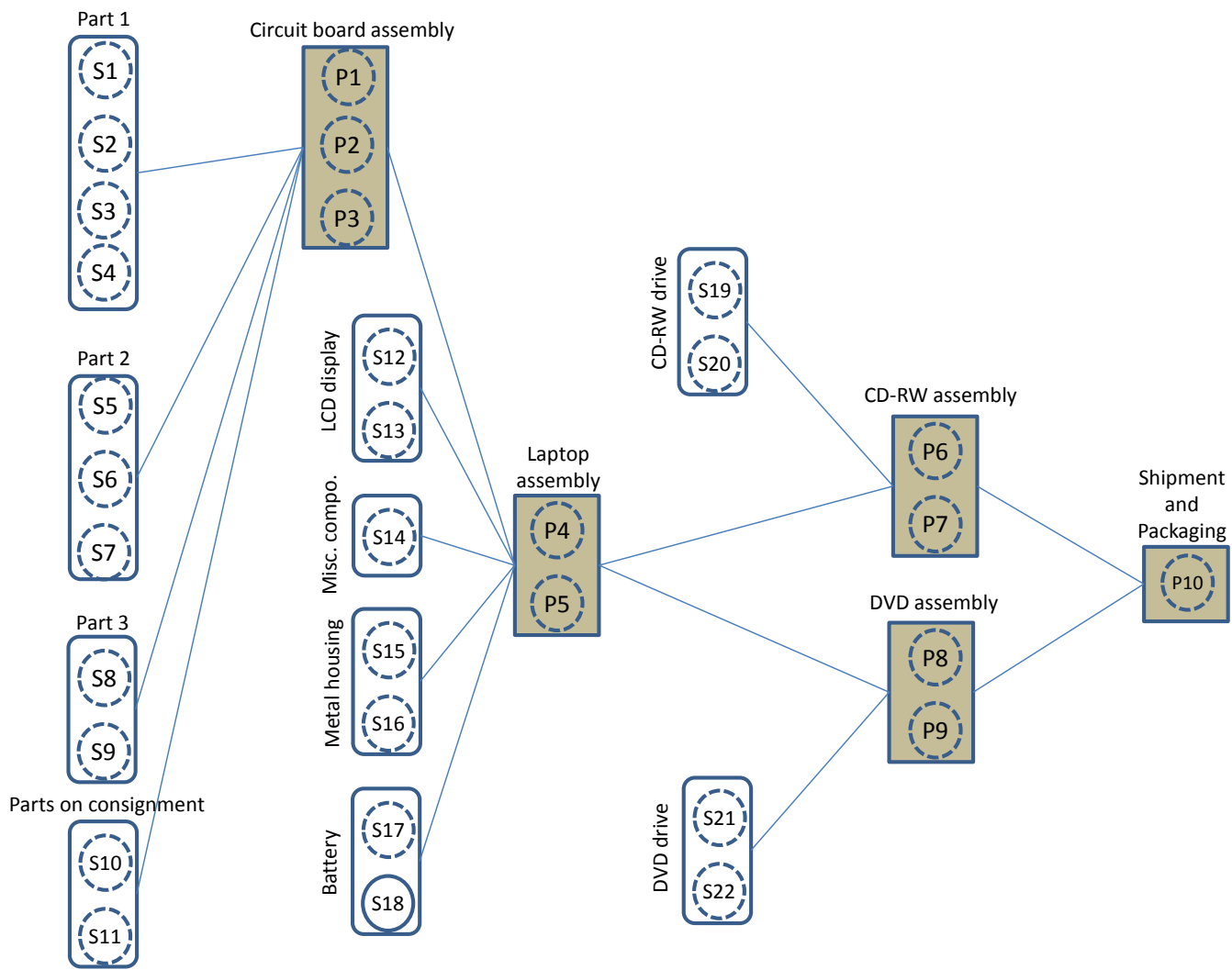

Figure 4: SC network for Laptops

As shown in Figure 4, the modified example has multiple options (in terms of suppliers and assemblers) to choose from at each network node when configuring the SC. For example, for part 1, the available sources are S1, S2, S3 and S4; and for the circuit board assembly, the available sources are P1, P2 and P3. Each of these alternative sources represents different values for lead times, costs and supply quantity.

The distance between two nodes, as an attribute to compute carbon emissions, has also been considered. However, it was assumed that road-based freight is the only transport option available. Table 1 illustrates the configuration dataset sample for suppliers for parts 1 and 2 only.

The data retrieval agent extracts raw production and sourcing information of relevant network entities such as suppliers and assemblers. This information is passed on to the knowledge acquisition agent to perform pre-processing of the raw product structure information. In pre- 
processing, the knowledge acquisition agent derives the fuzzy B-discernibility matrix from the raw information based on generalised fuzzy rough sets. The dataset for each node (SC entity) is divided into $70 \%$ for training and $30 \%$ for validation/testing. This helps in generalising the results from the classification system and to avoid over-fitting. The resulting matrix is then used to induct rule-based classifiers for each node in the SC network.

Table 1: Aggregated configuration data on suppliers for parts 1 and 2 (only)

\begin{tabular}{|c|c|c|c|c|c|c|c|}
\hline Stage & Node & Components & Supplier & Lead time & Quantity & Cost (\$) & $\begin{array}{c}\text { Distance } \\
\text { (Km) }\end{array}$ \\
\hline 1 & 1 & Part 1 & S1 & $1-10$ & 50 & 145 & 148.36 \\
\hline 1 & 1 & Part 1 & S1 & $11-20$ & 100 & 139 & 148.36 \\
\hline 1 & 1 & Part 1 & S1 & $21-40$ & 150 & 125 & 148.36 \\
\hline 1 & 1 & Part 1 & S2 & $1-5$ & 60 & 144 & 175.36 \\
\hline 1 & 1 & Part 1 & S2 & $6-25$ & 95 & 140 & 175.36 \\
\hline 1 & 1 & Part 1 & S2 & $26-40$ & 150 & 125 & 175.36 \\
\hline 1 & 1 & Part 1 & S3 & $1-25$ & 40 & 140 & 223.23 \\
\hline 1 & 1 & Part 1 & S3 & $26-35$ & 115 & 138 & 223.23 \\
\hline 1 & 1 & Part 1 & S3 & $36-40$ & 150 & 132 & 223.23 \\
\hline 1 & 1 & Part 1 & S4 & $1-15$ & 80 & 141 & 184.23 \\
\hline 1 & 1 & Part 1 & S4 & $16-40$ & 150 & 133 & 184.23 \\
\hline 1 & 2 & Part 2 & S5 & $1-5$ & 40 & 50 & 123.63 \\
\hline 1 & 2 & Part 2 & S5 & $6-9$ & 90 & 45 & 123.63 \\
\hline 1 & 2 & Part 2 & S5 & $10-12$ & 120 & 43 & 123.63 \\
\hline 1 & 2 & Part 2 & S5 & $13-15$ & 150 & 42 & 123.63 \\
\hline 1 & 2 & Part 2 & S6 & $1-7$ & 30 & 49 & 145.96 \\
\hline 1 & 2 & Part 2 & S6 & $8-10$ & 80 & 43 & 145.96 \\
\hline 1 & 2 & Part 2 & S6 & $11-15$ & 150 & 42 & 145.96 \\
\hline 1 & 2 & Part 2 & S7 & $1-10$ & 75 & 50 & 123.23 \\
\hline 1 & 2 & Part 2 & S7 & $11-15$ & 150 & 40 & 123.23 \\
\hline
\end{tabular}

Table 2: Prediction accuracy (percentage correctly classified) for all the nodes in the SC

\begin{tabular}{|c|l|c|}
\hline Node & $\begin{array}{c}\text { Component/process } \\
\text { description }\end{array}$ & $\begin{array}{c}\text { Fuzzy Rough Sets } \\
\text { Theory } \\
\text { (GFRS) }\end{array}$ \\
\hline 1 & Part 1 & 68.23 \\
\hline 2 & Part 2 & 67.9035 \\
\hline 3 & Part 3 & 82.243 \\
\hline 4 & Parts on consignment & 93.33 \\
\hline 5 & Circuit board assembly & 72.02 \\
\hline 6 & LCD display & 94.061 \\
\hline 7 & Miscellaneous components & 100.00 \\
\hline 8 & Metal housing & 89.074 \\
\hline 9 & Battery & 75.647 \\
\hline 10 & Laptop assembly & 71.1838 \\
\hline 11 & CD-RW drive & 99.17695 \\
\hline 12 & DVD drive & 86.104 \\
\hline 13 & CD-RW assembly & 80.939 \\
\hline
\end{tabular}




\begin{tabular}{|c|l|c|}
\hline 14 & DVD assembly & 83.07692 \\
\hline 15 & Shipment and Packaging & 100.00 \\
\hline
\end{tabular}

The knowledge representation agent runs the rule induction algorithm individually for each SC network node in a distributed manner (on training dataset) and the production rules generated are stored in the database at the end. The rules generated are tested with the help of the test dataset (30\% of the raw dataset). The results of the classification (i.e. percentage correctly classified for test dataset) for each node of the SC network are shown in Table 2. It should be noted that there is only one configuration option available for SC entity at nodes 7 and 15 . Therefore the classification is $100 \%$. On average, the accuracy of the classifiers used for all the nodes (excluding nodes 7 and 15) is 81.77. This means that the proposed classifier system is able to predict the configuration for SC entities accurately.

The rules generated are then stored in a production rules database. The configuration predictor agent retrieves information about the product orders from the customers and applies the rule-based classification to obtain suitable SC configurations. The customer orders for the two product variants (Laptop-CD and Laptop-DVD) are shown in Table 3. The results obtained after the application of stored production rules to each customer order is presented in Table 4. For each order, a suitable SC entity configuration is obtained to fulfil the customer order in a cost-effective and timely manner. From Table 4, it is clear that there is no one particular configuration of SC entities that suit all orders. The most common SC configuration (based on Table 4), however, is S4, S7, S9, S11, P1, S13, S14, S16, S17, P4, S20, S21, P6, P8 and P10.

Table 3: Customer orders for laptops

\begin{tabular}{|c|c|c|c|}
\hline ID & Product Type & $\begin{array}{c}\text { Lead } \\
\text { Time }\end{array}$ & Quantity \\
\hline 1 & Laptop-CD & 8 & 142 \\
\hline 2 & Laptop-CD & 56 & 136 \\
\hline 3 & Laptop-CD & 8 & 148 \\
\hline 4 & Laptop-CD & 37 & 140 \\
\hline 5 & Laptop-CD & 19 & 60 \\
\hline 6 & Laptop-CD & 26 & 9 \\
\hline 7 & Laptop-CD & 77 & 100 \\
\hline 8 & Laptop-CD & 59 & 131 \\
\hline 9 & Laptop-CD & 57 & 96 \\
\hline 10 & Laptop-CD & 68 & 35 \\
\hline 11 & Laptop-DVD & 44 & 137 \\
\hline 12 & Laptop-DVD & 57 & 43 \\
\hline 13 & Laptop-DVD & 7 & 135 \\
\hline 14 & Laptop-DVD & 61 & 94 \\
\hline 15 & Laptop-DVD & 20 & 57 \\
\hline 16 & Laptop-DVD & 8 & 125 \\
\hline 17 & Laptop-DVD & 22 & 27 \\
\hline 18 & Laptop-DVD & 40 & 7 \\
\hline 19 & Laptop-DVD & 47 & 147 \\
\hline 20 & Laptop-DVD & 80 & 41 \\
\hline
\end{tabular}


The SC configuration evaluator agent takes the results of the SC configuration (Table 4) and evaluates them against balking criteria based on the actual lead times required to match the customer's expected delivery time. Table 5 illustrates this evaluation, where order ID 1,3,4,5 for Laptop-CD and 11, 13, 15, 16 for Laptop-DVD is balked. Then the production cost per product is evaluated for the two cases: (i) when dynamic SC configuration is used, and (ii) when fixed common SC configuration is used. Table 5 shows the results of the SC evaluation process, where savings of \$7,337 (for Laptop-CD) and \$5,069.5 (for Laptop-DVD) can be achieved by dynamically configuring the SCs. The scenario where only sales profit and delivery time are used to evaluate the SC configuration is termed as scenario (i) and it would be used for comparisons against other scenarios (where carbon emissions is also considered).

Table 4: SC configuration results for the orders in scenario (i), where, $\mathrm{N} \_1$ represents node 1

\begin{tabular}{|c|c|c|c|c|c|c|c|c|c|c|c|c|c|c|c|}
\hline ID & N_1 & N_2 & N_3 & N_4 & N_5 & N_6 & N_7 & N_8 & N_9 & N_10 & $N_{-} 11$ & N_12 & N_13 & N_14 & N_15 \\
\hline 1 & S4 & S5 & S8 & S10 & P3 & S13 & S14 & S16 & S17 & $\mathrm{P} 4$ & S19 & S21 & P6 & & P10 \\
\hline 2 & S4 & S7 & S9 & S11 & $\mathrm{P} 1$ & S13 & S14 & S16 & S17 & P5 & S20 & S21 & P6 & & P10 \\
\hline 3 & S4 & S5 & S8 & S10 & P3 & $\mathrm{S} 13$ & S14 & S16 & S17 & $\mathrm{P} 4$ & S19 & S21 & P6 & & P10 \\
\hline 4 & S4 & S7 & S9 & S11 & P2 & $\mathrm{S} 13$ & S14 & S16 & S17 & P4 & S19 & S22 & P6 & & P10 \\
\hline 5 & S3 & S6 & S9 & S11 & P1 & S13 & S14 & S16 & S17 & P4 & S19 & S22 & P7 & & P10 \\
\hline 6 & S1 & S7 & S9 & S11 & $\mathrm{P} 1$ & $\mathrm{~S} 13$ & S14 & S16 & S17 & P4 & S19 & S21 & P6 & & P10 \\
\hline 7 & S2 & S7 & S8 & S11 & P2 & S12 & S14 & S16 & S17 & P4 & S20 & S21 & P7 & & P10 \\
\hline 8 & S2 & S7 & S9 & S11 & $\mathrm{P} 1$ & S13 & S14 & S16 & S17 & $\mathrm{P} 4$ & S20 & S21 & P6 & & P10 \\
\hline 9 & S2 & S7 & S9 & S11 & P1 & S13 & S14 & S16 & S17 & P4 & S20 & S21 & P6 & & P10 \\
\hline 10 & S1 & S7 & S9 & S11 & P2 & S13 & S14 & S16 & S17 & P5 & S20 & S21 & P7 & & P10 \\
\hline 11 & S4 & S7 & S9 & S11 & P1 & S13 & S14 & S16 & S17 & P5 & S20 & S21 & & P8 & P10 \\
\hline 12 & S2 & S7 & S9 & S11 & P1 & S13 & S14 & S16 & S17 & P4 & S20 & S21 & & P9 & P10 \\
\hline 13 & S4 & S5 & S9 & S11 & P3 & S13 & S14 & S16 & S17 & P4 & S19 & S22 & & P8 & P10 \\
\hline 14 & S2 & S7 & S8 & S11 & P3 & $\mathrm{S} 13$ & S14 & S16 & S17 & P4 & S20 & S21 & & P9 & P10 \\
\hline 15 & S3 & S5 & S9 & S11 & $\mathrm{P} 1$ & S13 & S14 & S16 & S17 & $\mathrm{P} 4$ & S19 & S21 & & P8 & P10 \\
\hline 16 & S4 & S5 & S9 & S10 & P3 & S13 & S14 & S16 & S17 & P4 & S19 & S21 & & P8 & P10 \\
\hline 17 & S1 & S7 & S9 & S11 & $\mathrm{P} 1$ & $\mathrm{~S} 13$ & S14 & S16 & S18 & P5 & S19 & S21 & & P8 & $\mathrm{P} 10$ \\
\hline 18 & S4 & S7 & S9 & S11 & P2 & $\mathrm{S} 13$ & S14 & S16 & S17 & P5 & S19 & S22 & & P8 & P10 \\
\hline 19 & S1 & S7 & S9 & S11 & $\mathrm{P} 1$ & $\mathrm{~S} 13$ & S14 & S16 & S17 & P4 & S20 & S21 & & P8 & P10 \\
\hline 20 & S1 & S7 & S9 & S11 & P2 & S12 & S14 & S15 & S17 & P4 & S20 & S21 & & P9 & P10 \\
\hline
\end{tabular}

The social cost of carbon emissions arising out of manufacturing and logistics activities is also used to configure SCs using the proposed framework. An average $\mathrm{CO}_{2}$ emission factor of $62 \mathrm{~g} \mathrm{CO}_{2} /$ tonne-km (CEFIC, 2011), used for activity-based $\mathrm{CO}_{2}$ emission estimations, is considered. This estimate was used for computing the transportation-related emissions. For the purposes of this paper, we have only considered road-based transport. Additionally, manufacturing-related emissions were calculated using an estimate of 61.1 tonnes of $\mathrm{CO}_{2}$ when a ton of product, by mass, is produced $\left(\mathrm{CO}_{2} \mathrm{List}\right.$, 2012). The weight of a laptop is considered to be $2.2078 \mathrm{Kg}$. The social cost of $\mathrm{CO}_{2}$ emissions is used in this study to estimate the reduction of net social welfare due to emissions. A recent study by Moore and Diaz (2015) estimates that one additional ton of $\mathrm{CO}_{2}$ emitted in 2015 reduces net social welfare by 
US $\$ 220$. This estimate is used to compute the dollar equivalent of the harm caused by $\mathrm{CO}_{2}$ emissions.

Table 5: Order results evaluation for scenario (i)

\begin{tabular}{|c|c|c|c|c|}
\hline ID & $\begin{array}{l}\text { Order } \\
\text { status }\end{array}$ & $\begin{array}{l}\text { Estimated Production } \\
\text { Cost based on Proposed } \\
\text { SC configuration } \\
\text { (\$, per product) }\end{array}$ & $\begin{array}{c}\text { Estimated Production } \\
\text { Cost based on most } \\
\text { common SC } \\
\text { configuration } \\
\text { (\$, per product) }\end{array}$ & $\begin{array}{c}\text { Savings } \\
\text { (\$) }\end{array}$ \\
\hline 1 & BALK & 1142 & 1147.5 & 781 \\
\hline 2 & OK & 1149.5 & 1147.5 & -272 \\
\hline 3 & BALK & 1142 & 1147.5 & 814 \\
\hline 4 & BALK & 1137.5 & 1147.5 & 1400 \\
\hline 5 & BALK & 1214.25 & 1240.5 & 1575 \\
\hline 6 & OK & 1237 & 1246 & 81 \\
\hline 7 & OK & 1180.25 & 1169.5 & -1075 \\
\hline 8 & $\mathrm{OK}$ & 1129.5 & 1147.5 & 2358 \\
\hline 9 & OK & 1149.5 & 1169.5 & 1920 \\
\hline 10 & OK & 1253 & 1246 & -245 \\
\hline \multicolumn{4}{|r|}{ Total } & 7,337 \\
\hline 11 & BALK & 1151.5 & 1147.5 & -548 \\
\hline 12 & $\mathrm{OK}$ & 1237.5 & 1244 & 279.5 \\
\hline 13 & BALK & 1143.5 & 1147.5 & 540 \\
\hline 14 & $\mathrm{OK}$ & 1165 & 1169.5 & 423 \\
\hline 15 & BALK & 1221.5 & 1240.5 & 1083 \\
\hline 16 & BALK & 1147 & 1151.5 & 562.5 \\
\hline 17 & OK & 1252 & 1246 & -162 \\
\hline 18 & OK & 1248 & 1246 & -14 \\
\hline 19 & OK & 1131.5 & 1147.5 & 2352 \\
\hline 20 & OK & 1230.5 & 1244 & 553.5 \\
\hline \multicolumn{4}{|r|}{ Total } & $5,069.5$ \\
\hline
\end{tabular}

The proposed framework is used again in the scenario where social cost from $\mathrm{CO}_{2}$ emission is used together with the production cost in evaluating SCs. This scenario is termed as scenario (ii). The results obtained from the SC configuration agent for scenarios (i) and (ii) are presented in Table 6. It is evident from Table 6 that although the production cost per product based on SC configuration is comparatively higher for scenario (ii) the combined cost is lower in scenario (ii). This is true for most of the orders. It can also be seen that the number of orders that are balking is lower in scenario (ii) when compared to scenario (i). 
Table 6: Comparison between scenarios (i) when production cost only, and (ii) combined production cost and social cost of $\mathrm{CO}_{2}$, is used for SC configuration

\begin{tabular}{|c|c|c|c|c|c|c|}
\hline & \multicolumn{3}{|c|}{ Scenario (i) } & \multicolumn{3}{c|}{ Scenario (ii) } \\
\hline ID & $\begin{array}{c}\text { Order } \\
\text { status }\end{array}$ & $\begin{array}{c}\text { Estimated } \\
\text { Production } \\
\text { Cost (\$, per } \\
\text { product) }\end{array}$ & $\begin{array}{c}\text { Estimated } \\
\text { Combined Cost } \\
\text { (\$, per product) }\end{array}$ & $\begin{array}{c}\text { Order } \\
\text { status }\end{array}$ & $\begin{array}{c}\text { Estimated } \\
\text { Production Cost } \\
\text { (\$, per product) }\end{array}$ & $\begin{array}{c}\text { Estimated } \\
\text { Combined Cost } \\
\text { (\$, per product) }\end{array}$ \\
\hline 1 & BALK & 1142 & 1228.80 & BALK & 1151.5 & 1214.45 \\
\hline 2 & OK & 1149.5 & 1233.06 & OK & 1139.5 & 1199.87 \\
\hline 3 & BALK & 1142 & 1232.05 & BALK & 1151.5 & 1217.53 \\
\hline 4 & BALK & 1137.5 & 1223.32 & OK & 1149 & 1210.47 \\
\hline 5 & BALK & 1214.25 & 1258.85 & OK & 1235.5 & 1254.16 \\
\hline 6 & OK & 1237 & 1253.85 & OK & 1249 & 1241.60 \\
\hline 7 & OK & 1180.25 & 1246.64 & OK & 1174.25 & 1216.19 \\
\hline 8 & OK & 1129.5 & 1210.31 & OK & 1139.25 & 1197.05 \\
\hline 9 & OK & 1149.5 & 1211.26 & OK & 1158.25 & 1198.21 \\
\hline 10 & OK & 1253 & 1284.02 & OK & 1250.5 & 1256.03 \\
\hline 11 & BALK & 1151.5 & 1234.11 & BALK & 1152 & 1214.38 \\
\hline 12 & OK & 1237.5 & 1270.91 & OK & 1246.5 & 1257.66 \\
\hline 13 & BALK & 1143.5 & 1224.61 & BALK & 1151.5 & 1212.85 \\
\hline 14 & OK & 1165 & 1223.98 & OK & 1173.25 & 1213.93 \\
\hline 15 & BALK & 1221.5 & 1262.37 & OK & 1235.5 & 1254.63 \\
\hline 16 & BALK & 1147 & 1222.53 & BALK & 1151.5 & 1207.72 \\
\hline 17 & OK & 1252 & 1276.58 & OK & 1249 & 1252.78 \\
\hline 18 & OK & 1248 & 1261.79 & OK & 1257 & 1250.58 \\
\hline 19 & OK & 1131.5 & 1219.15 & OK & 1152 & 1219.52 \\
\hline 20 & OK & 1230.5 & 1263.70 & OK & 1251.5 & 1261.59 \\
\hline
\end{tabular}

The comparative summary of the SC configuration for scenarios (i) and (ii) is presented in Table 7. It can be seen that balking percentage is reduced when production cost and social cost is considered for both of the products. In scenario (ii), the average social cost for $\mathrm{CO}_{2}$ emissions per product is significantly reduced (from 76.32 to 52.53 for Laptop-CD and from 68.91 to 48.77 for Laptop-DVD). The net reduction in social cost (per product) is $31.17 \%$ and +29.23. The unit production cost has slightly increased under scenario (ii). However, the production cost losses for the two products are $0.506 \%$ and $0.795 \%$ under scenario (ii). It can be seen that the production losses are less compared to the gain achieved through the reduction in social costs under scenario (ii). As such, the decision-makers need to consider both of these costs in determining the preferred SC configuration.

Table 7: SC configuration based on production costs and social costs of emissions

\begin{tabular}{|c|c|c|c|c|c|c|}
\hline & \multicolumn{3}{|c|}{ Laptop-CD } & \multicolumn{3}{c|}{ Laptop-DVD } \\
\cline { 2 - 7 } Scenario & $\begin{array}{c}\text { Balking } \\
\mathbf{( \% )}\end{array}$ & $\begin{array}{c}\text { Average } \\
\text { Production } \\
\text { Cost (\$, } \\
\text { product) }\end{array}$ & $\begin{array}{c}\text { Average } \\
\text { Social Cost } \\
\text { for Emission } \\
\text { (\$, product) }\end{array}$ & $\begin{array}{c}\text { Balking } \\
\mathbf{( \% )}\end{array}$ & $\begin{array}{c}\text { Average } \\
\text { Production } \\
\text { Cost (\$, } \\
\text { product) }\end{array}$ & $\begin{array}{c}\text { Average } \\
\text { Social Cost } \\
\text { for Emission } \\
\text { (\$, product) }\end{array}$ \\
\hline Scenario (i) & 40 & $1,154.41$ & 76.32 & 40 & $1,165.03$ & 68.91 \\
\hline Scenario (ii) & 20 & $1,160.25$ & 52.53 & 30 & $1,174.29$ & 48.77 \\
\hline
\end{tabular}




\section{Conclusions and Future Directions}

Considering the diverse challenges faced by manufacturing enterprises seeking to sustain their competiveness in a dynamic global business environment, we proposed an advanced agent-based analytics framework to aid their SC network configuration decisions. As we set out to develop an advance analytics framework to support robust configuration decisions we relaxed a number of assumptions used in the extant studies, which our framework was built on (i.e. Akanle and Zhang, 2008; Troung and Azadivar, 2005; Piramuttu, 2005). For instance, the proposed framework accounted for two product variants, multiple sourcing options at each network node, as well as multiple performance objectives. It also captured decisions that span the entire SC network simultaneously and, by implication, represented multiple network links. In effect, this paper contributes to SC configuration research by addressing most of the research gaps identified through the literature review (listed in Q4 of Fig. 1). The framework first generated alternative SC configurations that aligned with competitive priorities, which were then evaluated against SC-level performance metrics. This approach provides an opportunity for organisations to appreciate the impact of a range of factors in the broader decision environment on SC configuration decisions and make strategic choices aimed at leveraging or mitigating such impacts.

Results generated through the application of the test case demonstrated that the configuration choices made using the proposed framework can yield significantly superior financial gains and SC-wide performance outcomes. It was demonstrated that the methodological approach and the constituent knowledge acquisition and representation rules employed are capable of handling sophisticated configuration problems involving multiple product types, sourcing options and performance objectives. As such, the proposed analytics framework has the potential to serve as a more effective decision support tool that help manufacturing enterprises address the challenges associated with responding to changing competitive dynamics while accounting for nuances of the decision environment. Overall, we believe, the proposed framework responds well to the calls made in the literature in that it has demonstrated its efficacy in terms of generating alternative configurations that are feasible (i.e. capable of fulfilling required customer orders) and circumstantially optimal (i.e. capable of delivering desired performance outcomes under a given set of circumstances).

Building on the results of this study, we aim to validate the proposed framework using a fullscale empirical case study in future research. The agent-based modelling approach proposed in the paper is fairly generic and hence can be applied to real world examples with minimal modifications, for example, to input parameters and data. There is also a further opportunity for more comprehensively comparing the results of a full-scale empirical study against those of previous studies that have used other comparable methodological approaches. This may help identify areas for further improvements which will lead to establishing the superiority and generalisability of the proposed analytic framework in unequivocal terms.

\section{References:}

Acar, Y., Kadipasaoglu, S. and Schipperijn, P. (2010), A decision support framework for global supply chain modelling: an assessment of the impact of demand, supply and lead-time uncertainties on performance, International Journal of Production Research, Volume 48, Issue 11, pp. 3245-3268. 
Akanle, O. M. and Zhang, D. Z. (2008), Agent-based Model for Optimising Supply-chain Configurations, International Journal for Production Economics, 115 (2), pp. 444-460.

Beamon, B. M. (1998), Supply Chain Design and Analysis: Models and Methods, International Journal of Production Economics, 55, pp. 281-294.

Blackhurst, J., Wu, T. and O’Grady, P. (2005), PCDM: A Decision Support Modelling Methodology for Supply Chain, Product and Process Decision Decisions, Journal of Operations Management, 23, pp. 325-343.

Bose, R. (2009), Advanced analytics: opportunities and challenges, Industrial Management \& Data Systems Vol. 109 No. 2, pp. 155-172.

CEFIC, 2011, Guidelines for Measuring and Managing CO2 Emission from Freight Transport Operations, Issue 1 March 2011.

CO2List, 2012, Amounts of CO2 Released when Making \& Using Products, http://www.co2list.org/files/carbon.htm, Accessed 16 Sep 2015.

Cornelis, C., De Cock, M., Radzikowska, A., Fuzzy rough sets: from theory into practice, in: W. Pedrycz, A. Skowron, V. Kreinovich (Eds.), Handbook of Granular Computing, Wiley, 2008, pp. 533-552.

Cornelis, C., Jensen, R., Hurtado, G., Slezak, D., Attribute selection with fuzzy decision reducts, Inform. Sci. 180 (2) (2010) 209-224.

Dai, H, Lin, J. and Long, Q. (2914), A fractal perspective-based methodological framework for supply chain modelling and distributed simulation with multi-agent system, International Journal of Production Research, Vol. 52, No. 22, 6819-6840.

Fawcett S. E and Waller, M. A (2014), Supply Chain Game Changers-Mega, Nano, and Virtual Trends - And Forces That Impede Supply Chain Design, Journal of Business Logistics, 35(3): 157-164,

Huang, G.Q., Zhang, X.Y., Liang, L., Towards integrated optimal configuration of platform products, manufacturing processes, and supply chains, Journal of Operations Management, Volume 23, Issues 3-4, April 2005, Pages 267-290

Iannone, R., Miranda, S. and Riemma, S. (2007), Supply chain distributed simulation: An efficient architecture for multi-model synchronization, Simulation Modelling Practice and Theory 15, pp. 221-236.

Jensen, R. and Shen, Q. (2009a), New approaches to fuzzy-rough feature selection, IEEE Trans. Fuzzy Syst. 19 pp. 824-838.

Jensen, R., Cornelis, C., Shen, Q., (2009b) Hybrid fuzzy-rough rule induction and feature selection, in: IEEE International Conference on Fuzzy Systems (FUZZIEEE), 2009, pp. 1151-1156.

Long, Q. and Zhang, W. (2014), An integrated framework for agent based inventoryproduction-transportation modelling and distributed simulation of supply chains, Information Sciences, 227, 567-581. 
Meixell, M. J. and Gargeya V. B. (2005), Global supply chain design: A literature review and critique Transportation Research Part E 41, 531-550

Monostori, L, Váncza, J., Kumara, S.R.T., Agent-Based Systems for Manufacturing, CIRP Annals - Manufacturing Technology, Volume 55, Issue 2, 2006, Pages 697-720, ISSN 00078506.

Moore, F.C., Diaz, D.B., 2015. Temperature impacts on economic growth warrant stringent mitigation policy, Nature Climate Change, 5, 127-131.

Mortenson, M. J., Doherty, N. F. and Robinson, S. (2015), Operational research from Taylorism to Terabytes: A research agenda for the analytics age, European Journal of Operational Research, 241, pp. 583-595.

Mustafee, N., Taylor, S., Katsaliaki, K., Dwivedi, Y. and Williams, M. (2012), Motivations and barriers in using distributed supply chain simulation. Intl. Trans. in Op. Res., 19: 733751.

Olhager, J., Pashaei, P. and Sternberg, H. (2015) Design of global production and distribution networks: A literature review and research agenda, International Journal of Physical Distribution \& Logistics Management, Vol. 45 No. 1/2, pp. 138-158.

Pawlak, Z. , Rough sets, Int. J. Comp. Sci. 11 (1982) 341-356.

Piramuthu, S. (2005), Knowledge-based framework for automated dynamic supply chain configuration. European Journal of Operational Research, 165(1), pp. 219-230.

Qu, T. Huanga, G. Q., Cungb, V., Mangioneb, F., (2010). Optimal configuration of assembly supply chains using analytical target cascading. International Journal of Production Research, 48(23), pp.6883-6907.

Radzikowska, A. M., Kerre, E. E., 2002. A comparative study of fuzzy rough sets. Fuzzy Sets Syst. 126, 2 (March 2002), 137-155.

Shen, W., Hao, Q., Yoon, H.J. \& Norrie, D.H. (2006). Applications of agent-based systems in intelligent manufacturing: an updated review. Advanced Engineering Informatics, Vol. 20, 415-431

Terzi, S. and Cavalieri, S. (2004), Simulation in the supply chain context: a survey, Computers in Industry 53, 3-16

Truong, T. H and Azadivar, F (2005), Optimal design methodologies for configuration of supply chains, International Journal of Production Research, Vol. 43, No. 11, 2217-2236.

Wang, W. Y. C. and Chan, H. K. (2010), Supply Chain Planning and Configuration in the Global Arena: A Syncretic Perspective, International Journal of Production Economics, 127, pp. 211-214.

Zhang, L., You, X., Jiao, J. and Helo, P. (2009), Supply Chain Configuration with Coordinated Product, Process and Logistics Decisions: An Approach based on Petri Nets, International Journal of Production Research, 47(23), pp. 6681-6706.

Zhao, S.Y., Tsang, E.C.C., Chen, D.G., Wang, X.Z., Building a rule-based classifier - a fuzzy-rough set approach, IEEE Trans. Knowl. Data Eng. 22 (2010) 624-638. 